\title{
A spectral unit
}

\section{Giacomo Prando summarizes the troubled history of the radian, a unit with the odd property of appearing and disappearing seemingly at will in dimensional formulas.}

A few years after the introduction of the International System of Units (SI), the Italian physicist Eligio Perucca expressed enthusiasm about its "very authoritative answer" to an issue that had been "lying unsolved on the table of metrology for several decades" ${ }^{1}$. He was referring to the "explicit and official recognition" ${ }^{\prime \prime}$ of the dimension of the radian as the unit for plane angles. Perucca was fair enough to add that "introducing radians among the physical dimensions causes the scalp to itch severely" [the quotes in this paragraph were translated from Italian by the author]. And indeed, the successive development of the radian's status within the SI looks rather troubled.

One radian is defined in the SI as "the angle subtended at the centre of a circle by an arc that is equal in length to the radius" Its choice as natural unit of plane angles $\theta$ is related to the fact that, when $\theta$ is expressed in radians, the relation $\sin (\theta) \approx \theta$ is valid for small $\theta$ values.

The radian was introduced as supplementary unit of the SI based on the resolutions of the 11th Conférence Générale des Poids et Mesures (CGPM) in 1960. Following the 20th CGPM in 1995, the category of supplementary units was abolished and the radian was defined as a dimensionless derived unit. Seemingly, there are good mathematical grounds behind this decision. Angles are invariant under scale transformations - moreover, in a Taylor expansion of $\sin (\theta)$ for small $\theta$, terms with odd powers of $\theta$ are summed up, so that $\theta$ must be dimensionless. To make this fact explicit, the definition 1 $\operatorname{rad}=1 \mathrm{~m} \mathrm{~m}^{-1}$ used in the SI brochure reminds us of the ratio between the arc length and the radius length.

Digging through the literature shows that the situation is not that clear. Whether the plane angle is actually a dimensionless physical quantity or not has been debated for decades, and several proposals have been put forward to treat the plane angle as inherently dimensional. However, these

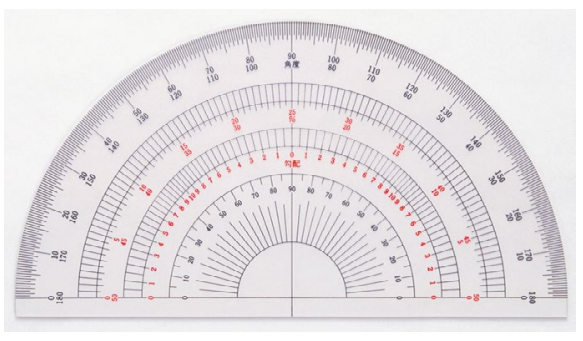

Credit: Imagenavi / Getty

all have a price to be paid, ranging from the use of different units for the radius and arc lengths to the distinction of trigonometric functions of angles and trigonometric functions of dimensionless numbers ${ }^{3-5}$. The subtleties of these issues are evident from the proceedings of the 26th CGPM in 2018. Here, a dedicated working group "focused on the pertinence of adding the radian as a new base unit of the SI and on the treatment of so-called 'dimensionless' quantities” but, “(a)fter many discussions, the group could not come to a consensus on any of these issues"6.

The current state of affairs leads inevitably to ghostly appearances and disappearances of the radian in the dimensional analysis of physical equations. This is well known to undergraduate students facing the formula for the characteristic angular frequency of the harmonic oscillator, who may wonder where the radian comes from. Another example comes from magnetic spectroscopy. The relaxation of the nuclear magnetization towards equilibrium is generally described by considering the effect of fluctuating random local magnetic fields on the statistical population of hyperfine levels ${ }^{7}$. Here, a crucial quantity is the correlation time, describing the average time between two fluctuations, and dimensional analysis would require that the units of this quantity are $\mathrm{s} \mathrm{rad}^{-1}$. This implies that relaxation rates and the probabilities of transitions between hyperfine levels per unit time should have rad s$^{-1}$ as their unit - however, the radian is often tacitly omitted. This discussion may sound pedantic but formulas for relaxation rates should be treated with great care in order to avoid gross numerical errors by forgotten factors $2 \pi$, and a stricter use of units can be of help in preventing such mistakes.

A related issue involves the Planck constant $h$. If the energy of the photon can be expressed in terms of frequency or angular frequency as $h \nu$ or $\hbar \omega$, respectively, then different units of $\nu$ and $\omega$ must be reflected in different units for $h$ and $\hbar$. Distinguishing cycles and radians seems natural, but the cycle is not an SI unit even though the hertz is. Furthermore, the current definition of the second derives from the duration of a number of periods of electromagnetic radiation, even though the cycle is not an accepted unit. It seems that, from whatever angle you look at the radian problem, circular arguments are unavoidable! If we are not ready yet to accept that $\hbar$ comes in dimensions of $\mathrm{J} \mathrm{s} \mathrm{rad}^{-1}$, we should at least convince ourselves that $h$ has units $\mathrm{J} \mathrm{Hz}^{-1}$ as a consistent - but currently unaccepted - consequence of the present structure of the SI.

\section{Giacomo Prando (D) $₫$}

Department of Physics, University of Pavia,

Pavia, Italy.

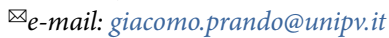

Published online: 4 August 2020

https://doi.org/10.1038/s41567-020-0997-3

\section{References}

1. Perucca, E. Fisica Generale e Sperimentale 8th edn, Preface (UTET, 1963).

2. The International System of Units 9th edn, 138 (BIPM, 2019); https://go.nature.com/3e8fLiA

3. Brownstein, K. R. Am. J. Phys. 65, 605 (1997).

4. Quincey, P., Mohr, P. J. \& Phillips, W. D. Metrologia 56, 043001 (2019).

5. Kalinin, M. I. Metrologia 56, 065009 (2019).

6. 26th Meeting of the CGPM - Proceedings 427 (BIPM, 2018); https://go.nature.com/3iIaPEE

7. Slichter, C. P. Principles of Magnetic Resonance Ch. 5 (Springer, 1990).

$\mathrm{m} e \mathrm{~A} \mathrm{~s} \mathrm{u} R \mathrm{~s} \mathrm{E}_{\mathrm{h}} \mathrm{s} \mathrm{R} \mu_{0} \varepsilon_{0} \alpha \mathrm{R}$

